\title{
The effect of postoperatively applied far-infrared radiation on pain and tendon-to-bone healing after arthroscopic rotator cuff repair: a clinical prospective randomized comparative study
}

\author{
Ji Young Yoon ${ }^{1}$, Joo Hyun Park ${ }^{2}$, Kwang Jin Lee ${ }^{3}$, Hyong Suk Kim ${ }^{4}$, Sung-Min Rhee ${ }^{5}$, and Joo Han Oh $^{6}$ \\ 'Department of Orthopaedic Surgery, National Police Hospital, Seoul, Korea \\ ${ }^{2}$ Department of Orthopaedic Surgery, Bundang Jesaeng Genenral Hospital, Seongnam, Korea \\ ${ }^{3}$ Department of Orthopaedic Surgery, Sun's Hospital, Seoul, Korea \\ ${ }^{4}$ Department of Orthopaedic Surgery, Incheon Sarang Hospital, Incheon, Korea \\ ${ }^{5}$ Department of Orthopeadic Surgery, Kyung Hee University College of Medicine, Kyung Hee University Hospital, Seoul, Korea \\ ${ }^{6}$ Department of Orthopaedic Surgery, Seoul National University College of Medicine, Seoul National University Bundang Hospital, Seongnam, Korea
}

Received March 23, 2020

Revised June 9, 2020

Accepted July 4, 2020

Handling Editor: Jin Woo Shin

\section{Correspondence}

Joo Han Oh

Department of Orthopaedic Surgery,

Seoul National University College of

Medicine, Seoul National University

Bundang Hospital, 82 Gumi-ro 173beon-

gil, Bundang-gu, Seongnam 13620,

Korea

Tel: +82-31-787-7197

Fax: $+82-31-787-4056$

E-mail: ohjh1@snu.ac.kr
Background: The effects of far-infrared radiation (FIR) on the treatment of rotator cuff diseases remains unknown. We evaluated the safety and efficacy of FIR after arthroscopic rotator cuff repair with regard to postoperative pain and healing.

Methods: This prospective randomized comparative study included 38 patients who underwent arthroscopic rotator cuff repair due to a medium-sized tear. Patients were randomly divided into the FIR or control group ( $n=19$ per group). In the FIR group, FIR with an FIR radiator started 1 week postoperatively for 30 minutes per session twice daily. It lasted until abduction brace weaning at 5 weeks postoperatively. We assessed pain using a pain visual analogue scale (pVAS) and measured the range of motion (ROM) of the shoulder at 5 weeks, and 3 and 6 months, postoperatively. The anatomical outcome was evaluated using magnetic resonance imaging at 6 months postoperatively.

Results: At 5 weeks postoperatively, the average pVAS score was lower in the FIR group than in the control group $(1.5 \pm 0.8$ vs. $2.7 \pm 1.7 ; P=0.019)$. At 3 months postoperatively, the average forward flexion was higher in the FIR group $\left(151.6^{\circ} \pm\right.$ $15.3^{\circ}$ vs. $132.9^{\circ} \pm 27.8^{\circ} ; P=0.045$ ), but there was no significant difference at 6 months postoperatively. There was no significant difference in healing failure between the groups $(P=0.999)$.

Conclusions: FIR after arthroscopic rotator cuff repair could be an effective and safe procedure to reduce postoperative pain, thereby facilitating rehabilitation and better ROM in the early postoperative period.

Key Words: Far-infrared Radiation; Pain Visual Analog Scale; Postoperative Pain; Range of Motion; Rehabilitation; Rotator Cuff Repair; Rotator Cuff Tears; Shoulder Pain.

\section{INTRODUCTION}

The incidence of rotator cuff tears is increasing rapidly, mainly due to the aging population and advancements in radiological diagnosis. Tears are reported in approximately $30 \%$ of those aged $\geq 60$ years and nearly $60 \%$ of those (c) This is an open-access article distributed under the terms of the Creative Commons Attribution Non-Commercial License (http://creativecommons.org/licenses/by-nc/4.0/), which permits unrestricted non-commercial use, distribution, and reproduction in any medium, provided the original work is properly cited.

(C) The Korean Pain Society, 2020
Author contributions: Ji Young Yoon: Writing/manuscript preparation; Joo Hyun Park: Investigation; Kwang Jin Lee: Investigation; Hyong Suk Kim: Investigation; Sung-Min Rhee: Investigation; Joo Han Oh: Supervision. 
aged $\geq 80$ years $[1,2]$. Arthroscopic rotator cuff repair has become a common surgical technique for treating rotator cuff tears, and as it is minimally invasive, it may have the additional benefits of reducing postoperative pain and improving early functional recovery [3]. Additionally, there is recent growing interest in various methods for improving functional recovery of patients after arthroscopic shoulder surgery, one of them being the use of far-infrared radiation (FIR) [4].

FIR is an electromagnetic wave, which is also emitted from the sun, and has wavelengths between 5.6 and 25.0 $\mu \mathrm{m}$ [5]. Depending on its wavelength, infrared radiation can be divided into the following three categories: nearinfrared radiation $(0.8-1.5 \mu \mathrm{m})$, middle-infrared radiation $(1.5-5.6 \mu \mathrm{m})$, and FIR $(5.6-1,000 \mu \mathrm{m})$ [5]. FIR has three biological effects, including radiation, resonance, and thermal effects [6]. FIR carries energy that is perceived as heat by thermo-receptors on the surrounding skin and can penetrate up to $4 \mathrm{~cm}$ beneath the skin [7]. Moreover, it can resonate with water and organic molecules within the body [8]. According to Wien law, the dominant wavelength of radiation emitted by a body at $35^{\circ} \mathrm{C}$ is $9.4 \mu \mathrm{m}$. This explains why the human body easily absorbs FIR between 4 and $16 \mu \mathrm{m}$ [9]. The FIR radiator used generates a 2-25 $\mu \mathrm{m}$ wavelength similar to the wavelength of the human body. The human body of an adult consists of $55 \%-60 \%$ water. FIR interacts with water molecules, causing a thermal reaction that raises the temperature of the tissue. The human body responds to this phenomenon by expanding blood vessels. Subsequently, blood circulation increases, more oxygenated blood reaches soft tissue, and at the same time, it stimulates the removal of accumulated toxins [8].

The FIR effect has been demonstrated by animal studies in rats, which resulted in increased nutrient supply to tissues, accelerated tissue repair, improved waste removal, and elevated pain thresholds $[5,10]$. Wong et al. [6] reported that FIR promoted local circulation and improved capillary flow after total knee arthroplasty. Subsequently, FIR may increase tissue oxygenation and improve healing by eliminating chronic inflammation, reducing pain and swelling, relieving muscle spasms, inducing relaxation of musculotendinous structures, and eventually relieving symptoms [5,6]. In orthopedic fields, FIR may be useful for wound healing and analgesia [6]. However, no comparative study has been performed to date that investigates the use of FIR after arthroscopic rotator cuff repair. Moreover, no studies have looked at how FIR may affect postoperative pain-relief, functional recovery, and rotator cuff healing during the tissue healing phase.

In this prospective randomized comparative study, we aimed to evaluate the safety and efficacy of FIR after ar- throscopic rotator cuff repair, based on the hypothesis that FIR can improve postoperative pain, functional recovery, and healing effects after surgery.

\section{MATERIALS AND METHODS}

\section{Cohort}

This prospective randomized study was conducted between June 2017 and January 2019. Its protocol was approved by the Institutional Review Boards of Seoul National University Bundang Hospital (No. E-1704-390-004) of the senior authors' institution. Written informed consent was obtained from all patients prior to surgery. We included patients aged between 20 and 80 years with a rotator cuff tear of more than $1 \mathrm{~cm}$ and less than $3 \mathrm{~cm}$. We excluded patients with (1) shoulder arthritis, (2) a large to massive rotator cuff tear, (3) an isolated subscapularis tear, (4) revision, (5) rheumatoid arthritis or other connective tissue disease, (6) a history of infection, (7) complex regional pain syndrome, (8) a preoperative hepatic or renal impairment, (9) uncontrolled diabetes mellitus or hypertension, (10) allergic skin lesions, (11) human immunodeficiency virus positive, and (12) women of reproductive age who could not undergo FIR. During the study period, we analyzed 38 patients and divided them randomly into the FIR $(\mathrm{n}=19)$ and control $(\mathrm{n}=19)$ groups (Fig. 1).

\section{Application protocol of FIR}

In the FIR group, FIR with FIR radiator (Aladdin- $\mathrm{H}^{\circledR}$; Taerim Medical Co. Ltd., Seongnam, Korea) was started 1 week after the operation, and lasted until the patient was weaned off abduction brace, which was approximately 5 weeks postoperatively. It was applied on the operated shoulder for 30 minutes per session twice daily. The control group did not receive FIR, and the other rehabilitation interventions were performed in the same manner as those of the FIR group. The FIR radiator was applied at a distance of $30-35 \mathrm{~cm}$ from the patient's skin, and patients themselves marked on a checklist whether FIR was applied to them (Fig. 2).

\section{Surgical procedures and perioperative management}

All procedures were performed under general anesthesia by the senior author (J.H.O), with the patient in the lateral decubitus position, using three arthroscopic portals (anterior, lateral, and posterior). After glenohumeral inspection, subacromial decompression was performed to remove 


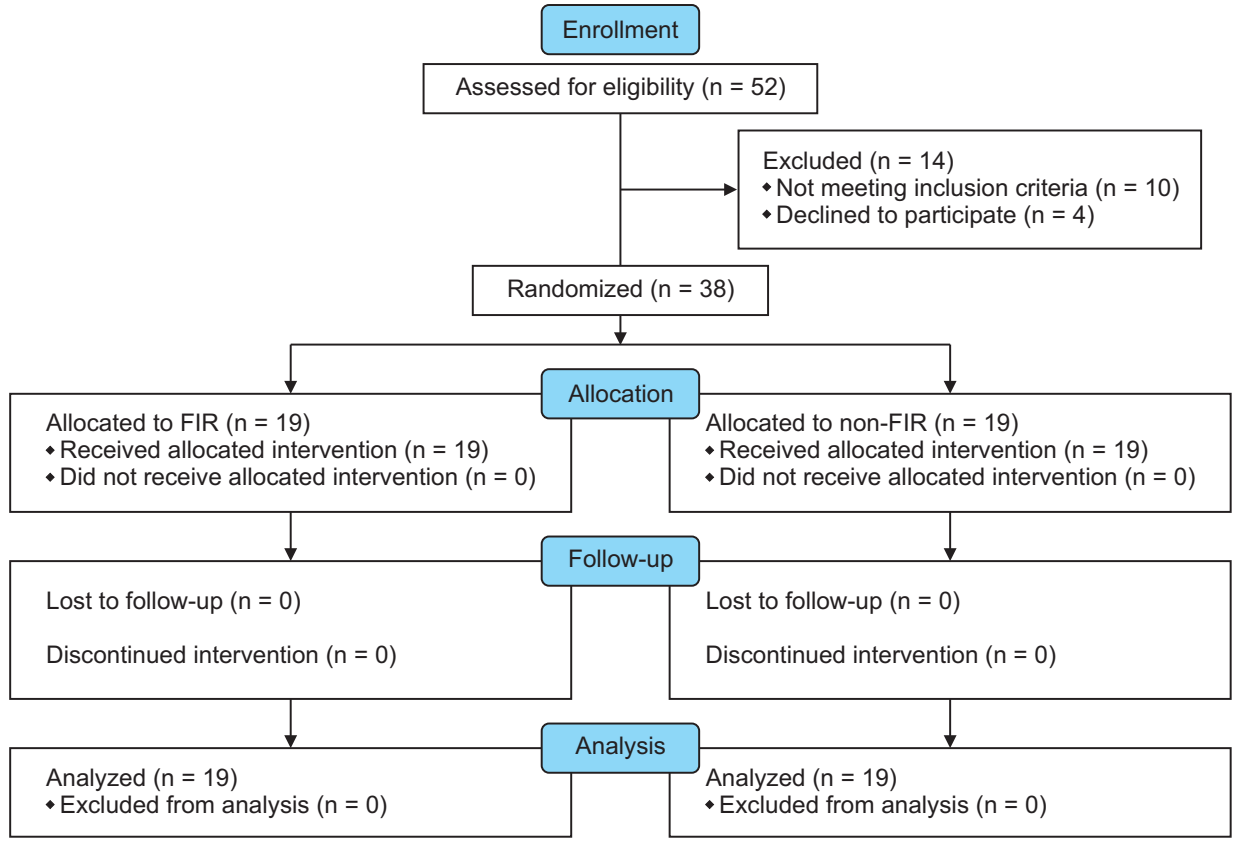

Fig. 1. Consolidated Standards of Reporting Trials flow diagram of the study is shown. FIR, far-infrared radiation.

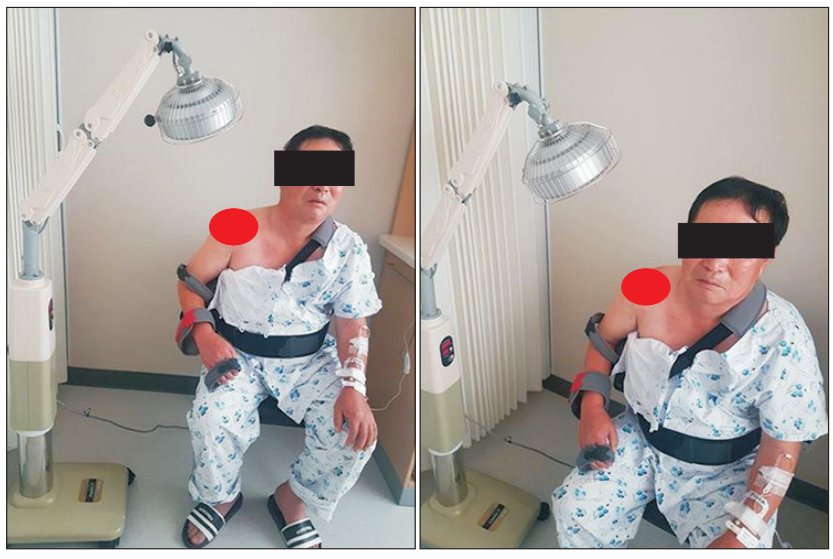

Fig. 2. Far-infrared radiation (FIR) with an FIR radiator: wave length 2-25 $\mu \mathrm{m}$ (Aladdin- $\mathrm{H}^{\circledR}$; Taerim Medical Co. Ltd., Seongnam, Korea).

the inflamed bursal tissue. Acromioplasty was conducted with a motorized burr in almost all patients. After footprint preparation was performed to expose the bleeding bony surface with a ring curette, rasp, and shaver, the anteroposterior and retraction tear length was measured with a calibrated probe. Rotator cuff repair was performed using the double row suture bridge technique or modified Mason-Allen technique with several suture anchors [11].

\section{Rehabilitation}

The postoperative rehabilitation for arthroscopic rotator cuff repair was the same in both groups. After surgery, an ice pack was applied to the operation site for 5 days for all patients. Postoperative pain management was administered to all patients, using the same oral pain medication, such as tramadol, up to 2 weeks postoperatively. To see only the effects of FIR itself, immobilization was maintained using an abduction brace for 5 weeks, for all patients. Shoulder shrugging, active elbow flexion/extension, active forearm supination/pronation, and active hand/ wrist motion were encouraged immediately after surgery. Passive range of motion (ROM) was not allowed during the brace-wearing periods. After weaning off the abduction brace, active-assisted and active-ROM exercises were encouraged, and muscle strengthening exercises began 10-12 weeks after surgery. All sporting activities were allowed at 6 months after surgery. Rehabilitation was co-supervised by the Department of Rehabilitation and Orthopedic Surgery.

\section{Clinical outcomes}

The primary endpoint was a visual analogue scale for pain (pVAS) at 5 weeks postoperatively, ranging from 0-10 (0, no pain; 10 , the worst pain). The pVAS was measured in all patients at each follow-up visit. The secondary endpoints were pVAS and ROM at 3 and 6 months postoperatively, and functional scores at 6 months postoperatively. ROM was measured using a goniometer with the fixed scapular position in 3 planes, including forward flexion (FF), external rotation (ER) at the side, and internal rotation (IR) at the back. FF was measured as the angle between the arm and thorax with the elbow fully extended. ER at the side was measured as the angle between the forearm and thorax with the upper arm in adduction and the elbow in $90^{\circ}$ flexion. IR at the back was measured by the vertebral level that the tip of the patient's thumb could reach while 
Table 1. Preoperative Demographic Data of Patients

\begin{tabular}{lccc}
\hline \multicolumn{1}{c}{ Variable } & FIR group $(\mathrm{n}=19)$ & Control group $(\mathrm{n}=19)$ & $P$ value \\
\hline Age $(\mathrm{yr})$ & $62.8 \pm 7.5$ & $60.8 \pm 8.5$ & 0.232 \\
Sex $($ male/female) & $10 / 9$ & $7 / 12$ & 0.328 \\
Height $(\mathrm{m})$ & $1.6 \pm 0.1$ & $1.6 \pm 0.1$ & 0.114 \\
Weight $(\mathrm{kg})$ & $67.0 \pm 9.3$ & $65.9 \pm 13.4$ & 0.403 \\
Tear retraction $(\mathrm{mm})$ & $15.9 \pm 6.3$ & $16.2 \pm 6.5$ & 0.722 \\
Tear AP diameter $(\mathrm{mm})$ & $14.8 \pm 4.0$ & $13.4 \pm 4.1$ & 0.259 \\
pVAS (points) & $5.5 \pm 2.4$ & $4.9 \pm 2.3$ & 0.407 \\
ASES score & $56.8 \pm 18.9$ & $55.5 \pm 19.0$ & 0.839 \\
SST score & $5.4 \pm 3.3$ & $3.5 \pm 3.7$ & 0.084 \\
Range of motion & & & \\
$\quad$ Forward flexion $\left({ }^{\circ}\right)$ & $157.4 \pm 10.5$ & $148.2 \pm 17.9$ & 0.162 \\
External rotation at the side $\left({ }^{\circ}\right)$ & $51.6 \pm 15.4$ & $49.7 \pm 14.6$ & 0.678 \\
Internal rotation at the back $($ level) & \\
\hline
\end{tabular}

Qualitative data were expressed as counts and percentages, and qualitative variables were expressed as the mean \pm standard deviation.

FIR: far-infrared radiation, AP: anteroposterior, pVAS: pain visual analogue scale, ASES: American Shoulder and Elbow Surgeons, SST: Simple Shoulder Test.

a Measured by the vertebral level that the patient was able to reach with the thumb and numbered serially as 1 to 12 for the 1 st to 12 th thoracic vertebrae, 13 to 17 for the 1st to 5th lumbar vertebrae, and 18 for any level below the sacral vertebrae.

in a sitting position. For convenience of analysis, authors numbered the vertebral levels serially, which are as follows: 1-12 for the 1st-12th thoracic vertebrae, and 13-17 for the 1st-5th lumbar vertebrae [12].

The functional outcome scores such as the American Shoulder and Elbow Surgeons (ASES) score and Simple Shoulder Test (SST) were also evaluated preoperatively and at 6 months postoperatively [13,14]. The ROM and functional scores were measured by a single researcher (K.S.J) who was blinded to the current study. Additionally, the third endpoint was the tendon-to-bone healing rate of the repaired rotator cuff at 3 months postoperatively, via ultrasound, and at 6 months postoperatively, via magnetic resonance imaging (MRI) [15]. The tendon-to-bone healing rate of the repaired rotator cuff was evaluated as the integrity of the repaired rotator cuff tendon [15].

\section{Statistical analysis}

All statistical analyses were performed using the Statistical Analysis System statistical software package, ver. 9.4 (SAS Institute, Cary, NC, USA). A $P$ value $<0.05$ was considered statistically significant. Categorical variables were compared using the chi-square test. For continuous variables, the Mann-Whitney $U$-test was used for comparisons between two groups.

Power analysis was performed to determine the sample size, and the statistical power of the study was calculated by G-Power software ver. 3.1.9.2 (Kiel, Germany). Using the postoperative pVAS average of $3.65 \pm 0.21$ (FIR group) and $4.05 \pm 0.39$ (control group) based on the data reported in the literature [6], we determined that a sample size of 30 patients (15 patients per group) was necessary to achieve a power (1-beta) of $90 \%$ at a significant level (alpha) of $5 \%$. Taking the dropout rate of $20 \%$ into account, the total sample size should be 19 patients per group. Thus, 38 patients who underwent arthroscopic rotator cuff tear surgery were enrolled in the current study.

\section{RESULTS}

Each patient was given a self-checklist to record the exact time and site of the irradiation throughout the treatment period. We confirmed their data, and all patients answered that they complied with the protocol well.

Demographic data, including preoperative pVAS, showed no difference between the FIR and control groups (Table 1). The pVAS at 5 weeks postoperatively, which was the primary outcome, was $1.5 \pm 0.8$ in the FIR group, which was statistically significantly lower than $2.7 \pm 1.7$ in the control group ( $P=0.019$, Table 2$)$.

For the secondary outcomes, pVAS at 3 and 6 months postoperatively were not different between the two groups. The pVAS at 3 months postoperatively was $2.1 \pm$ 0.9 in the FIR group, which was not significantly different from $2.7 \pm 1.9$ in the control group $(P=0.344$, Table 3$)$. The pVAS at 6 months postoperatively was $0.7 \pm 1.3$ in the FIR group, which was not statistically different from $0.6 \pm 1.0$ in the control group $(P=0.928$, Table 4$)$. At 3 months after surgery, the average FF was $151.6^{\circ} \pm 15.3^{\circ}$ in the FIR group, which was significantly higher than $132.9^{\circ} \pm 27.8^{\circ}$ in the control group ( $P=0.045$, Table 3 ), but there was no significant difference in the FF at 6 months postoperatively. 
Table 2. pVAS Score and Range of Motion at 5 Weeks Postoperatively

\begin{tabular}{lccc}
\multicolumn{1}{c}{ Variable } & FIR group $(\mathrm{n}=19)$ & Control group $(\mathrm{n}=19)$ & $P$ value \\
\hline pVAS (points) & $1.5 \pm 0.8$ & $2.7 \pm 1.7$ & $0.019 *$ \\
Range of motion & & & $79.5 \pm 23.9$ \\
$\quad$ Forward flexion $\left({ }^{\circ}\right)$ & $84.2 \pm 20.0$ & $23.4 \pm 12.0$ & 0.243 \\
$\quad$ External rotation at the side $\left({ }^{\circ}\right)$ & $22.6 \pm 9.2$ & $17.3 \pm 1.7$ & 0.911 \\
Internal rotation at the back $\left(\right.$ level) ${ }^{a}$ & $17.7 \pm 0.6$ & 0.910
\end{tabular}

Qualitative data were expressed as counts and percentages, and qualitative variables were expressed as the mean \pm standard deviation. pVAS: pain visual analogue scale, FIR: far-infrared radiation.

$* P<0.05$ statistically significant.

${ }^{a}$ Measured by the vertebral level that the patient was able to reach with the thumb and numbered serially as 1 to 12 for the 1 st to 12 th thoracic vertebrae, 13 to 17 for the 1 st to 5 th lumbar vertebrae, and 18 for any level below the sacral vertebrae.

Table 3. pVAS Score and Range of Motion at 3 Months Postoperatively

\begin{tabular}{lccc}
\hline \multicolumn{1}{c}{ Variable } & FIR group $(n=19)$ & Control group $(n=19)$ & $P$ value \\
\hline pVAS (points) & $2.1 \pm 0.9$ & $2.7 \pm 1.9$ & 0.344 \\
Range of motion & & & \\
Forward flexion $\left(^{\circ}\right)$ & $151.6 \pm 15.3$ & $132.9 \pm 27.8$ & $0.045^{*}$ \\
External rotation at the side $\left(^{\circ}\right)$ & $38.4 \pm 10.8$ & $35.3 \pm 17.0$ & 0.682 \\
Internal rotation at the back (level) $)^{a}$ & $12.7 \pm 2.4$ & $13.1 \pm 3.1$ & 0.396 \\
\hline
\end{tabular}

Qualitative data were expressed as counts and percentages, and qualitative variables were expressed as the mean \pm standard deviation. pVAS: pain visual analogue scale, FIR: far-infrared radiation.

$* P<0.05$ statistically significant.

${ }^{a}$ Measured by the vertebral level that the patient was able to reach with the thumb and numbered serially as 1 to 12 for the 1 st to 12 th thoracic vertebrae, 13 to 17 for the 1 st to 5 th lumbar vertebrae, and 18 for any level below the sacral vertebrae.

Table 4. Clinical Outcomes at 6 Months Postoperatively

\begin{tabular}{|c|c|c|c|}
\hline Variable & FIR group $(n=19)$ & Control group $(n=19)$ & $P$ value \\
\hline pVAS (points) & $0.7 \pm 1.3$ & $0.6 \pm 1.0$ & 0.928 \\
\hline ASES score & $88.2 \pm 10.2$ & $90.7 \pm 9.3$ & 0.352 \\
\hline SST score & $9.9 \pm 1.5$ & $10.1 \pm 1.8$ & 0.647 \\
\hline \multicolumn{4}{|l|}{ Range of motion } \\
\hline Forward flexion $\left({ }^{\circ}\right)$ & $154.5 \pm 8.3$ & $155.0 \pm 10.1$ & 0.988 \\
\hline External rotation at the side $\left(^{\circ}\right)$ & $61.6 \pm 12.6$ & $61.6 \pm 18.6$ & 0.858 \\
\hline Internal rotation at the back (level) $)^{a}$ & $9.8 \pm 1.7$ & $9.1 \pm 1.3$ & 0.299 \\
\hline
\end{tabular}

Qualitative data were expressed as counts and percentages, and qualitative variables were expressed as the mean \pm standard deviation.

FIR: far-infrared radiation, pVAS: pain visual analogue scale, ASES: American Shoulder and Elbow Surgeons, SST: Simple Shoulder Test.

${ }^{a}$ Measured by the vertebral level that the patient was able to reach with the thumb and numbered serially as 1 to 12 for the 1 st to 12 th thoracic vertebrae, 13 to 17 for the 1st to 5th lumbar vertebrae, and 18 for any level below the sacral vertebrae.

Other ROMs were not significantly different between the two groups at 3 and 6 months postoperatively. In addition, functional outcome measurements at 6 months postoperatively, including ASES and SST scores, were not significantly different between the two groups $(P=0.352$ for ASES score and $P=0.647$ for SST, Table 4).

Regarding the third outcome, which was the tendon-tobone healing rate of the rotator cuff at 3 months postoperatively, via ultrasound, and at 6 months postoperatively, via MRI, there was no significant difference in the healing failure between the two groups. At 3 months postoperatively, no healing failure was found in either group using ultrasound, but there were two healing failure cases $(10.5 \%)$ in the FIR group and one healing failure case $(5.3 \%)$ in the control group $(P=0.999)$ at 6 months postoperatively, via MRI.

The potential adverse effects of FIR, such as skin burn, rash, infection, wound dehiscence, hypersensitivity reaction, and body temperature elevation, did not occur in our patients. Moreover, there were no other perioperative complications noted in any patient in either group. 


\section{DISCUSSION}

This prospective randomized comparative study evaluated the safety and efficacy of FIR after rotator cuff repair, and the current data demonstrated that the pVAS was significantly lower in the FIR group than in the control group at 5 weeks postoperatively. At 3 months postoperatively, the average FF was significantly higher in the FIR group than in the control group; however, other variables including the pVAS were not significantly different. At 6 months postoperatively, there were no differences in the pVAS, $\mathrm{ROM}$, functional scores, or healing failure rate between the two groups, and adverse events from FIR were not observed in the FIR group.

In the medical field, FIR is usually used for patients with lymphedema and vascular disease $[8,16]$. Its surgical application has been actively investigated for plastic surgery and oral-maxillofacial surgery $[17,18]$. In plastic surgery, FIR has been proven to induce wound healing by increasing skin microcirculation in rats undergoing flap treatment [17]. In oral-maxillofacial surgery, FIR has been proven to aid wound healing after tooth extraction $[18,19]$. The application of FIR is still unchartered territory in the orthopedic field. However, we hope that our research will be a cornerstone in proving the efficacy and safety of its clinical use. Further studies into FIR are required not only to evaluate its effectiveness in the clinical setting, such as increasing microcirculation, but also to determine its latent mechanisms.

Pain is an important factor experienced by patients after elective surgery and usually occurs due to the injury of nerve endings and soft tissue damage $[6,20]$. According to previous studies, $77 \%-98 \%$ of patients experienced postoperative pain, and up to $50 \%$ of this same group experienced moderate pain $[6,21]$. Severe pain adversely affects patients' biologic, physical, and psychological status [20].

Additionally, arthroscopic rotator cuff repair causes more severe pain than arthroscopic surgery of other joints owing to bone resection, extensive removal of bursal tissue, insertion of a suture anchor, and soft tissue distension from irrigation fluid [22]. This postoperative pain usually interferes with early rehabilitation, adversely affecting surgical outcomes and extending hospitalization, leading to decreased patient satisfaction [23]. Proper pain control after arthroscopic rotator cuff repair can prevent serious complications by reducing hospitalization, thereby achieving early ROM and muscle strength [24]. In our study, pVAS was significantly lower in the FIR group than the control group 5 weeks postoperatively $(P=0.019)$. This indicates that FIR significantly improved pain-relief, particularly in the early postoperative period. This corresponds with the regional irradiation achieved by FIR, penetrating almost 4 $\mathrm{cm}$ beneath the skin, and causing local capillary dilatation due to transmission of thermal energy [7]. As a result, it increases nutrient supply to specific tissue, promotes tissue repair, improves elimination of waste, and raises patients' pain threshold [5,6]. Moreover, Wong et al. [6] reported that patients who received FIR after total knee arthroplasty have reduced interleukin-6 (IL-6), indicating an antiinflammatory effect. IL- 6 is secreted by injured tissue, and plays a central role in the immune response [25]. IL-6 level correlates not only with inflammation, but also with pain-relief [6]. Reducing pain facilitates postoperative rehabilitation of patients who have undergone arthroscopic rotator cuff repair [24].

As mentioned previously, proper pain control after arthroscopic rotator cuff repair can lead to early ROM achievements $[24,26]$. In our study, FF in ROM was significantly higher in the FIR group than in the control group at 3 months postoperatively $(P=0.045)$, which supports the preceding sentence. Recent studies have shown that FIR reduces wound healing time and provides soothing effects and pain-relief following standard medical wound treatment [18]. However, in the current study, pVAS at 3 and 6 months after surgery was not significantly different. Moreover, except for FF at 3 months postoperatively, ROM was not significantly different between the two groups at 3 and 6 months after the operation. Supporting these results, the functional outcome at 6 months after surgery, including ASES and SST scores, were also not significantly different ( $P=0.352$ and $P=0.647$, respectively). The results of these findings suggest that FIR for 5 weeks after surgery may help in reducing early postoperative pain, thereby promoting early ROM at 3 months postoperatively. It is necessary to conduct a subsequent long-term study of pVAS, ROM, and functional outcomes, while continuing to apply FIR until 3-6 months postoperatively to determine if this further reduces pain and facilitates postoperative rehabilitation in patients who had undergone arthroscopic rotator cuff repair.

Martin [27] explained that wound healing involves the following three steps: inflammation, granulated tissue formation, and tissue remodeling. In the first inflammatory phase, transforming growth factor (TGF)- $\beta 1$ and plateletderived growth factor are released from macrophages. In the second granulation phase, TGF- $\beta 1$ activates fibroblasts to produce extracellular matrix proteins, such as collagen and fibronectin. Finally, in the third tissue remodeling phase, TGF- $\beta 1$ enables fibroblast migration, which is a critical step in wound healing. Toyokawa et al. [5] reported that FIR can influence skin wound healing by stimulating secretion of TGF- $\beta 1$ or by activating fibroblasts independent of skin blood flow and skin temperature. They proved that the number of migrating fibroblasts expressing TGF- 
$\beta l$ was significantly higher in the FIR group. Secretion of TGF- $\beta 1$ by platelets and macrophages is thought to be enhanced by FIR; as a result, TGF- $\beta 1$ stimulates fibroblast migration. Consequently, collagen production was also increased in the FIR group. The production of collagen fibers due to FIR-induced activation of fibroblasts has been considered as a possible mechanism for the promotional effect of FIR on wound healing [5].

However, in our study, the healing failure rate was not statistically different $(P=0.999)$. Taking into account the same postoperative rehabilitation process after FIR application, subjects of this study were patients with relatively small tears $(1-3 \mathrm{~cm})$. Therefore, healing is expected to be satisfactory without FIR [2]. Subsequent studies should include patients with larger rotator cuff tears to determine whether FIR would enhance rotator cuff healing. Furthermore, longer periods of FIR application would enhance rotator cuff healing. Considering that the rotator cuff healing process usually continues up to 6 months after surgical repair, it would be better to verify whether FIR lasting for 3-6 months postoperatively would enhance rotator cuff healing. More research is needed with respect to the regularity and durations of radiation, and the effective wavelength range $[5,27,28]$.

There are several strengths in this study. First, this is the first prospective randomized comparative clinical trial for evaluating the efficacy and safety of FIR after rotator cuff repair. Until now, there have been several studies investigating the clinical application of FIR, mainly for skin or lymphedema $[8,29,30]$. Therefore, the results of this preliminary analysis seem to be promising and could be applied especially to other orthopedic fields. Second, the current study included homogenous patients in terms of tear size, operation time, difficulties and extent of surgery, and rehabilitation; thus, we can estimate the effects of FIR more objectively. However, our work was subject to several limitations as well. First, this study had a relatively short-term follow-up. Although adverse effects such as hypersensitivity reaction and re-tear of the repaired rotator cuff are known to occur in the early postoperative period, long-term follow-up is needed to evaluate the efficacy and safety of FIR. Second, as we have discussed above, patients with larger tears should be included, and several options of FIR application, such as duration, regularity, and wavelength, should be investigated in a future large-scale and long-term clinical studies to verify the healing effect of FIR. Third, at 3 months after surgery, only FF, except ER and IR, is statistically significant. So, it is a little difficult to conclude that the effect of FIR is on ROMs improvement. Lastly, it is impossible to ensure patient compliance, although we have provided a checklist to record the exact time and site of irradiation throughout the treatment pe- riod.

In conclusion, FIR after arthroscopic rotator cuff repair could be an effective and safe procedure to reduce postoperative pain, thereby facilitating postoperative rehabilitation and resulting in better ROM in the early postoperative period. Further studies and clinical trials are needed with respect to the regularity and durations of radiation, effective wavelength range, and rotator cuff healing.

\section{CONFLICT OF INTERST}

No potential conflict of interest relevant to this article was reported.

\section{FUNDING}

This study was supported by SeongNam Industry Promotion agency (www.snip.or.kr).

\section{ORCID}

Ji Young Yoon, https://orcid.org/0000-0002-5496-9584 Joo Hyun Park, https://orcid.org/0000-0002-9450-2807 Kwang Jin Lee, https://orcid.org/0000-0001-8844-2716 Hyong Suk Kim, https://orcid.org/0000-0001-6283-8392 Sung-Min Rhee, https://orcid.org/0000-0002-7087-4219 Joo Han Oh, https://orcid.org/0000-0002-4962-3175

\section{REFERENCES}

1. Yamaguchi K, Ditsios K, Middleton WD, Hildebolt CF, Galatz LM, Teefey SA. The demographic and morphological features of rotator cuff disease. A comparison of asymptomatic and symptomatic shoulders. J Bone Joint Surg Am 2006; 88: 1699704.

2. Kukkonen J, Kauko T, Virolainen P, Äärimaa V. The effect of tear size on the treatment outcome of operatively treated rotator cuff tears. Knee Surg Sports Traumatol Arthrosc 2015; 23: $567-72$.

3. Baumgarten KM, Vidal AF, Wright RW. Rotator cuff repair rehabilitation: a level I and II systematic review. Sports Health 2009; 1: 125-30.

4. Oh JH, Yoon JY. Various regimens for the functional recovery after arthroscopic shoulder surgery. J Korean Orthop Assoc 2020; 55: 103-16.

5. Toyokawa H, Matsui Y, Uhara J, Tsuchiya H, Teshima S, Nakanishi $\mathrm{H}$, et al. Promotive effects of far-infrared ray on fullthickness skin wound healing in rats. Exp Biol Med (May- 
wood) 2003; 228: 724-9.

6. Wong CH, Lin LC, Lee HH, Liu CF. The analgesic effect of thermal therapy after total knee arthroplasty. J Altern Complement Med 2012; 18: 175-9.

7. Vatansever F, Hamblin MR. Far infrared radiation (FIR): its biological effects and medical applications. Photonics Lasers Med 2012; 4: 255-66.

8. Li K, Zhang Z, Liu NF, Feng SQ, Tong Y, Zhang JF, et al. Efficacy and safety of far infrared radiation in lymphedema treatment: clinical evaluation and laboratory analysis. Lasers Med Sci 2017; 32: 485-94.

9. Dominguez-Vidal A, Kaun N, Ayora-Cañada MJ, Lendl B. Probing intermolecular interactions in water/ionic liquid mixtures by far-infrared spectroscopy. J Phys Chem B 2007; 111: 4446-52.

10. Akasaki Y, Miyata M, Eto H, Shirasawa T, Hamada N, Ikeda $\mathrm{Y}$, et al. Repeated thermal therapy up-regulates endothelial nitric oxide synthase and augments angiogenesis in a mouse model of hindlimb ischemia. Circ J 2006; 70: 463-70.

11. Oh JH, Oh CH, Kim SH, Kim JH, Yoon JP, Jung JH. Clinical features of partial anterior bursal-sided supraspinatus tendon (PABST) lesions. J Shoulder Elbow Surg 2012; 21: 295-303.

12. Oh JH, Kim SH, Lee HK, Jo KH, Bin SW, Gong HS. Moderate preoperative shoulder stiffness does not alter the clinical outcome of rotator cuff repair with arthroscopic release and manipulation. Arthroscopy 2008; 24: 983-91.

13. Kocher MS, Horan MP, Briggs KK, Richardson TR, O'Holleran J, Hawkins RJ. Reliability, validity, and responsiveness of the American Shoulder and Elbow Surgeons subjective shoulder scale in patients with shoulder instability, rotator cuff disease, and glenohumeral arthritis. J Bone Joint Surg Am 2005; 87: 2006-11.

14. Beaton DE, Richards RR. Measuring function of the shoulder. A cross-sectional comparison of five questionnaires. J Bone Joint Surg Am 1996; 78: 882-90.

15. Codsi MJ, Rodeo SA, Scalise JJ, Moorehead TM, Ma CB. Assessment of rotator cuff repair integrity using ultrasound and magnetic resonance imaging in a multicenter study. J Shoulder Elbow Surg 2014; 23: 1468-72.

16. Lin CC, Chang CF, Lai MY, Chen TW, Lee PC, Yang WC. Farinfrared therapy: a novel treatment to improve access blood flow and unassisted patency of arteriovenous fistula in hemodialysis patients. J Am Soc Nephrol 2007; 18: 985-92.

17. Yu SY, Chiu JH, Yang SD, Hsu YC, Lui WY, Wu CW. Biological effect of far-infrared therapy on increasing skin microcir- culation in rats. Photodermatol Photoimmunol Photomed 2006; 22: 78-86.

18. Lin YH, Li TS. The application of far-infrared in the treatment of wound healing: a short evidence-based analysis. J Evid Based Complementary Altern Med 2017; 22: 186-8.

19. Arany PR, Nayak RS, Hallikerimath S, Limaye AM, Kale AD, Kondaiah P. Activation of latent TGF-betal by low-power laser in vitro correlates with increased TGF-betal levels in laser-enhanced oral wound healing. Wound Repair Regen 2007; 15: 866-74.

20. Breivik H. Postoperative pain management: why is it difficult to show that it improves outcome? Eur J Anaesthesiol 1998; 15: 748-51.

21. Owen H, McMillan V, Rogowski D. Postoperative pain therapy: a survey of patients' expectations and their experiences. Pain 1990; 41: 303-7.

22. Rhee SM, Chung NY, Jeong HJ, Oh JH. Subacromial local anesthetics do not interfere with rotator cuff healing after arthroscopic repair. Am J Sports Med 2018; 46: 1097-105.

23. Fredrickson MJ, Ball CM, Dalgleish AJ. Analgesic effectiveness of a continuous versus single-injection interscalene block for minor arthroscopic shoulder surgery. Reg Anesth Pain Med 2010; 35: 28-33.

24. Warrender WJ, Syed UAM, Hammoud S, Emper W, Ciccotti MG, Abboud JA, et al. Pain management after outpatient shoulder arthroscopy: a systematic review of randomized controlled trials. Am J Sports Med 2017; 45: 1676-86.

25. Regulation of the acute phase and immune responses: interleukin-6. Ann NY Acad Sci 1989; 557: 1-583.

26. Oh JH, Kim WS, Kim JY, Gong HS, Rhee KY. Continuous intralesional infusion combined with interscalene block was effective for postoperative analgesia after arthroscopic shoulder surgery. J Shoulder Elbow Surg 2007; 16: 295-9.

27. Martin P. Wound healing--aiming for perfect skin regeneration. Science 1997; 276: 75-81.

28. Yu W, Naim JO, Lanzafame RJ. Expression of growth factors in early wound healing in rat skin. Lasers Surg Med 1994; 15: 281-9.

29. Masuda A, Koga Y, Hattanmaru M, Minagoe S, Tei C. The effects of repeated thermal therapy for patients with chronic pain. Psychother Psychosom 2005; 74: 288-94.

30. Ou SM, Hu FH, Yang WC, Lin CC. Far-infrared therapy as a novel treatment for encapsulating peritoneal sclerosis. Am J Gastroenterol 2014; 109: 1957-9. 